\title{
DIGITAL TWIN FOR FATIGUE ANALYSIS
}

\author{
Amaury Chabod* \\ Nicolas Baron \\ HBK France PRENSCIA, 3 chemin de la Dîme, 95700 Roissy-en-France \\ *amaury.chabod@hbkworld.com
}

\begin{abstract}
The main design parameters that impact the fatigue of components are geometry, material and loading. Simulation with Finite Element Analysis (FEA) and tests on a vibrating table are often used to understand the dynamic behaviour of components and thus validate those items.

Accelerated tests are used for the mission profile and test definition, as described in GAM-EG-13, MIL-STD-810F and RTCA DO-160E. The shock response spectrum (SRS) and the extreme response spectrum (ERS) allow for a comparison of the power spectrum density (PSD) and the acceleration factor applied in terms of fatigue severity through the fatigue damage spectrum (FDS). In addition, the hypothesis of linear damage accumulation enables the combination of several events for specifying a mission profile. Ultimately, the mission profile, which represents a usage that might span over several years, can be reduced to a shorter duration with a damage extraction technique. This is particularly useful for the definition of vibrating table specifications.

An advantage of the virtual vibrating table is the reduction of the number of prototypes and the understanding of failure modes. To achieve this objective, finite element analysis in the frequency domain (harmonic analysis) is used and the structural stress response is evaluated with a PSD loading. A statistical model of rainflow allows assessing the damage on the components. The presentation also shows the effects of the damping factor on damage results. To achieve accurate results and define a Digital Twin, the correlation between test results and the finite element analysis is fundamental. Experimental modal analysis, based on the measured acceleration responses, helps to validate calculated modal frequencies and to assess the damping for each mode. This study shows the importance and the sensitivity on damping of the structural response, and in turn on fatigue.
\end{abstract}

Keywords: Fatigue analysis, digital twin method, modal analysis, test-FEA correlation

Article Category: Research Article 


\section{INTRODUCTION}

The need to reduce design costs, time-to-market and carbon dioxide emission implies not only decreasing the number of prototypes and tests duration but also requires accurate simulation modelling.

The main parameters taken into account in building a fatigue model are geometry, material and loading. Simulating vibration fatigue is well established, improving precision may be achieved by finely analyzing tests results.

In this paper, it will be explored why it is important to use strain gage and accelerometers measurements to achieve a better correlation between Finite Element Analysis (FEA) and a real prototype on a test bench. Correlation concerns any quasi-static loading but proves to be more difficult for a dynamic model. For the latter, Experimental Modal Analysis (EMA), Operational Deformed Shape (ODS) and Modal Assurance Criterion (MAC) are used to match resonances frequencies and modal deformed shapes, both qualitatively and quantitatively. In addition, we will see the importance of accurately measuring the damping ratio for dynamic and vibration fatigue analysis.

Then, we will describe how accelerated tests are used for the mission profile and the test definition, as described in GAM-EG-13, MIL-STD-810F and RTCA DO-160E, to obtain a better description of the PSD (Power Spectrum Density) loading input for shaker tables, thus reducing the test duration.

Finally, after having built the foundations of our digital twin, we will see how to conduct a vibration fatigue analysis in the time domain and in the frequency domain and how to improve physical representativity compared to a real shaker table.

\section{FATIGUE ROADMAP}

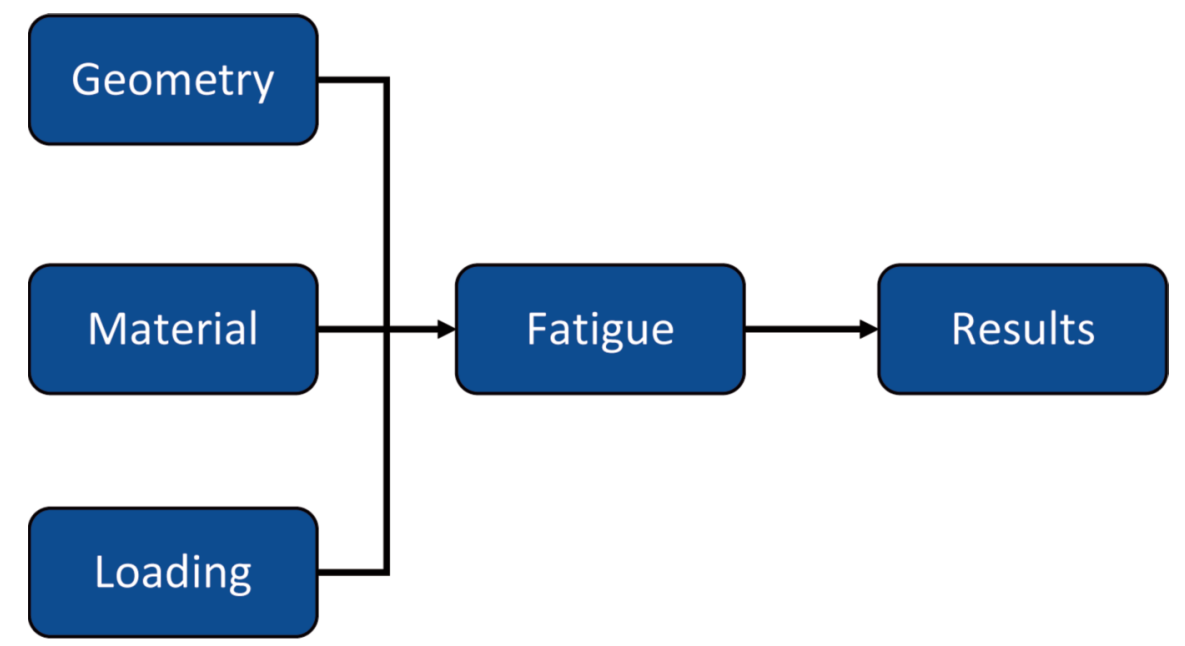

Fig. 1. Fatigue Roadmap.

\section{GEOMETRY: TEST/FEA CORRELATION}

Correlation of FEA with the test is a major step towards building a digital twin. Many questions must be answered regarding the representativity of the FE model, the boundary 
conditions, the stiffness or the stress response. In this section, we will see how virtual sensors help to check if the FE model sees the same level of acceleration or strain as the physical component does. Experimental modal analysis will be used to correlate resonances frequencies and to measure damping.

\subsection{Correlation with strain gages}

A tubular beam model was built. The beam was clamped at $z=0$ and the punctual mass $\mathrm{m}$ was positioned at a distance $z=L_{\text {mass }}$.

Table 1. Beam model definition.

\begin{tabular}{|c|c|c|c|}
\hline Basis & $\mathrm{r}$ & 8 & $\mathrm{~mm}$ \\
\hline Height & $\mathrm{R}$ & 9 & $\mathrm{~mm}$ \\
\hline Surface & S & 53.40 & $\mathrm{~mm}^{2}$ \\
\hline Inertia & $\mathrm{I}$ & 1936 & $\mathrm{~mm}^{4}$ \\
\hline Length & $\mathrm{L}$ & 362 & $\mathrm{~mm}$ \\
\hline $\begin{array}{c}\text { Length at mass } \\
\text { position }\end{array}$ & $\mathrm{L}$ mass & 348 & $\mathrm{~mm}$ \\
\hline Mass & $\mathrm{m}$ & 277 & $\mathrm{~g}$ \\
\hline Density & $\mathrm{r}$ & $2.70 \mathrm{E}-09$ & $\mathrm{t} / \mathrm{m}^{3}$ \\
\hline Acceleration & $\mathrm{g}$ & $9.81 \mathrm{E}+03$ & $\mathrm{~mm} / \mathrm{s}^{2}$ \\
\hline
\end{tabular}

The simplicity of this model enables analytical calculations with the standard beam formula [2]:

$$
\text { Max bending stress at radius } R: \sigma=\frac{M_{f}}{I} R
$$

Inertia for tube ( $R$ : outer radius, $r$ : inner radius): $I=\pi\left(R^{4}-r^{4}\right)$

Bending moment ( $z$ : axial position of gage): $M_{f}=\frac{1}{2} \rho g S(L-z)^{2}+m g\left(L_{\text {mass }}-z\right)$

Equation 1. Beam equations for stress calculation.

Bending moment includes the mass distributed from the tube and the punctual mass. The same model was built with FE model with the Abaqus software. 

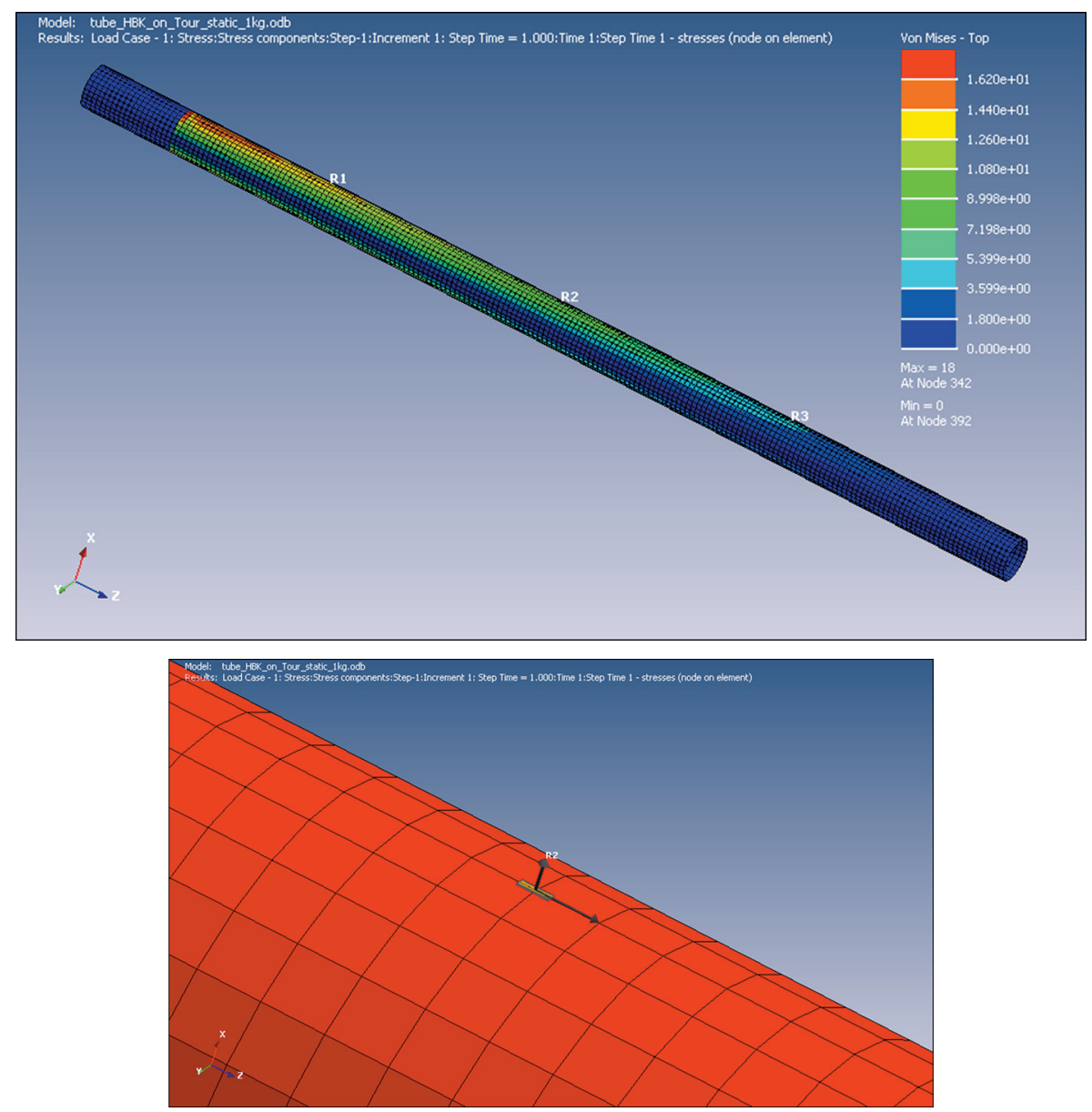

\begin{tabular}{|c|c|}
\hline Resistive strain gages & $\begin{array}{c}\text { Distance } \mathrm{z} \text { from } \\
\text { clamping }\end{array}$ \\
\hline R1 & 62 \\
\hline R2 & 162 \\
\hline R3 & 262 \\
\hline
\end{tabular}

Fig. 2. Virtual strain gages position on FE model.

Strain gages are positioned on the FE model in the same position and orientation as during the test, thanks to the virtual strain gage capabilities of nCode DesignLife software.

The microstrain correlation between FE and test is good. We can observe that the FE model is a little more conservative due to the rigid clamping assumption. The test results are from -7 to $-14 \%$ lower in magnitude. What if we perform a pseudo-fatigue life analysis in these quasi-static results with the same material? No experimental fatigue tests were carried out to produce such results; life was obtained from the microstrain and the Basquin curve $\left(\sigma=A \cdot N^{b}\right.$, with $A=2000 \mathrm{MPa}$ and $\left.b=-0.2\right)$ for comparison purposes. 


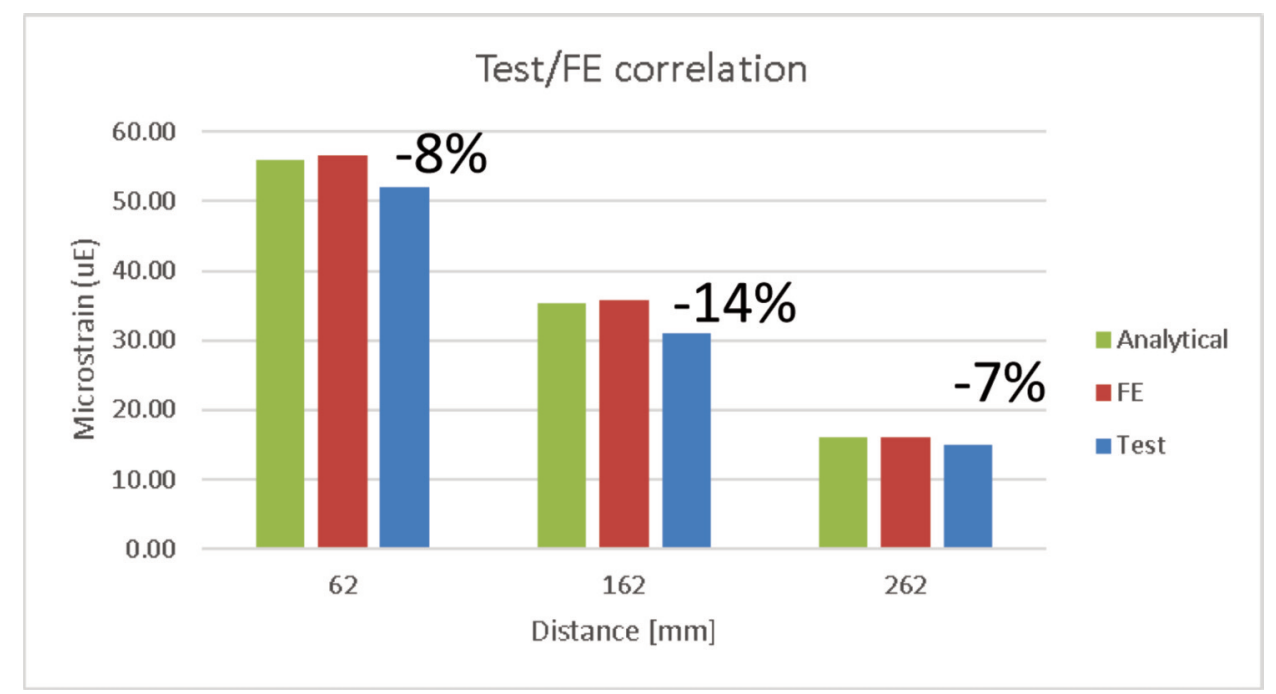

Fig. 3. Microstain comparison between analytic (green), FE (red) and test (blue) [1].

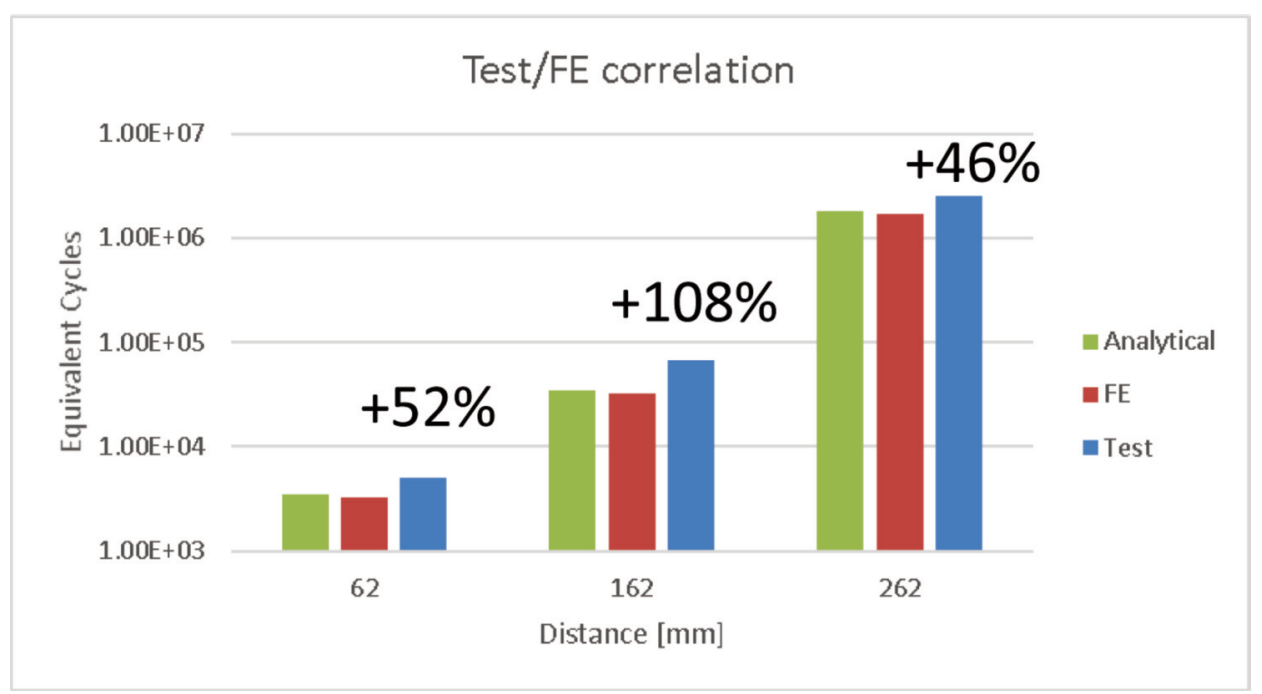

Fig. 4. Equivalent life obtained (cycles) [1].

This graph shows that small differences in microstrain in test $(-7 \%$ to $-14 \%)$ generate comparatively large differences in the damage results $(+46 \%$ to $+108 \%)$. It reveals that errors in strains or stresses are magnified in life results; this is, indeed, due to the power law defined by the Basquin formulation. At this stage, the conclusion is that before doing any fatigue analysis, it is important that a proper fit of the strain/stress between FE and test be achieved.

\subsection{Correlation with accelerometers}

The model was tested to check for the natural frequencies, including the analysis of test accelerometers data resulting from hammer impacts. 
For a better understanding, natural frequencies for a simple clamped beam were calculated analytically and by FE modal analysis.

$$
\begin{gathered}
v_{i}=\frac{1}{2 \pi} \alpha_{i}^{2} \sqrt{\frac{E}{\rho}} \frac{1}{L^{2}} \sqrt{\frac{I}{S}} \\
\alpha_{i} \text { are roots of equation: } \cos \left(\alpha_{i}\right) \cosh \left(\alpha_{i}\right)=-1
\end{gathered}
$$

( $E$ : Young Modulus, $r$ : density, $L$ : length of beam, $I$ : inertia, $S$ : cross section of beam)

Equation 2. Natural frequencies for clamped beam [5].

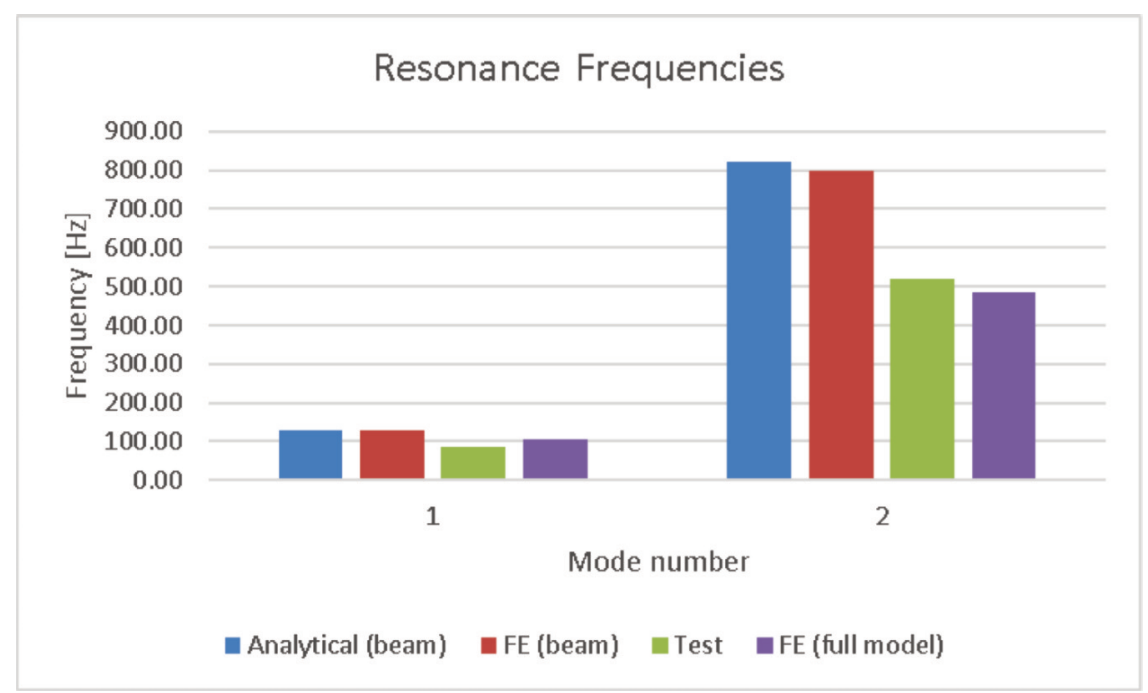

Fig. 5. Test/FEA correlation for natural frequencies [1].

Analytical natural frequencies (in blue) are close to the simple FEA model (in red). Comparison with the test bench (in green) drives us to consider a more realistic clamping with much lower stiffness and to construct a full FEA model (in purple). Doing so, the correlation is much closer to reality in terms of magnitude of resonance frequencies.

In the pictures below, the modal deformed shape is plotted for the first and the second modes, for both the FE simple beam and the full FE model.

Experimental Modal Analysis (EMA) is capable of automatically identifing natural frequencies using the Frequency Response Function obtained from hammer testing. Operational Deformed Shape (ODS) and Modal Assurance Criterion (MAC) matrix complete the validation of the modal deformed shape accuracy with testing. 

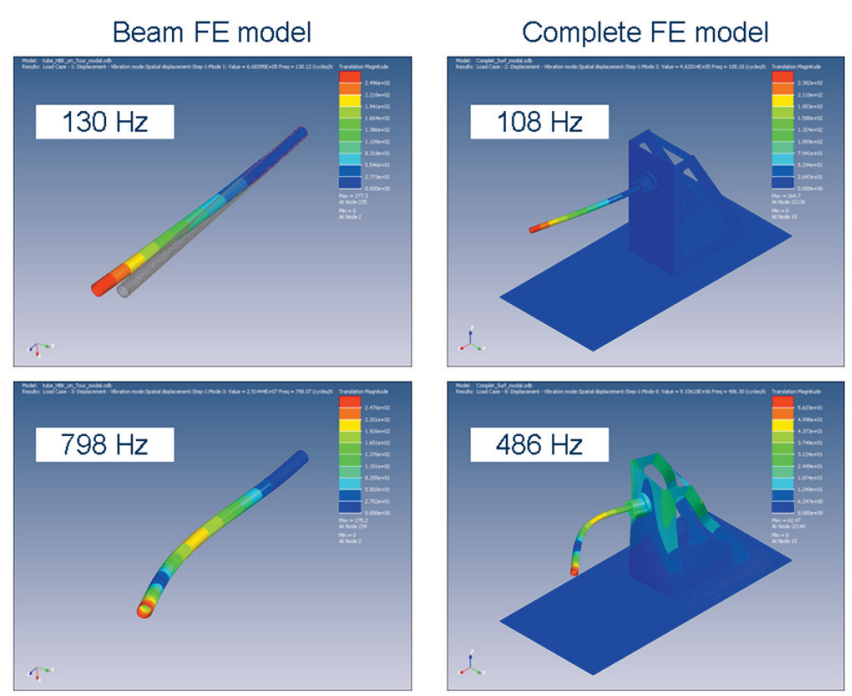

Fig. 6. Comparison for natural frequencies between simple clamped beam and full model with test bench support, mode \#1 (top) and mode \#2 (bottom) [1].

Additionally, the damping ratio is directly extracted from the test by EMA, which enables accurate simulating of even the dynamic model response. Indeed, matching resonance frequencies and the deformed shapes of the modal analysis is not sufficient to get results close to reality. A numerical example below shows how the simulated life is impacted by changing the damping ratio from $2 \%$ to $1.5 \%$ : simulated life is divided by two.
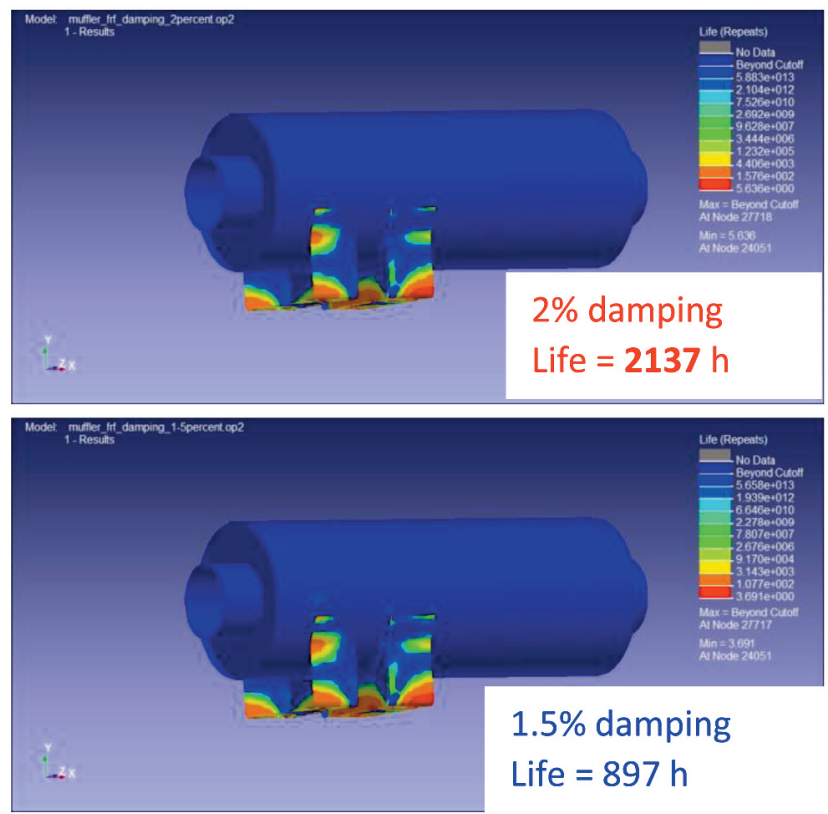

Fig. 7. Comparison of fatigue life obtained with $2 \%$ and $1.5 \%$ damping ratio [4].

It is really the damping ratio that drives the magnitude of the stress response at resonance frequencies. As for static analysis, accurate stress response prediction is an important step to be taken before performing any fatigue analysis. 


\section{LOADING: POWER SPECTRUM DENSITY (PSD)}

Describing customer usage, variability and all real-life situations in service requires considering a huge amount of data. But only by doing so one can define another major input of fatigue analysis, which is loading and the specification to apply to the component /system. In case of the dynamic analysis, spectra such as Power Spectrum Density are used for describing random, stationary, ergodic and gaussian loads. Some relevant questions concern how to compare current service measurements with the standards, how to merge several usage assessments into a single PSD, how to reduce the test duration from years of in-service loading to a few months of tests in a design project.

A well-established framework addresses these issues arriving at an elegant and simple model (NATO STANAG 4370 (AECTP-240)/ UK DefStan 00-35/Mil-Std-810G) [3].

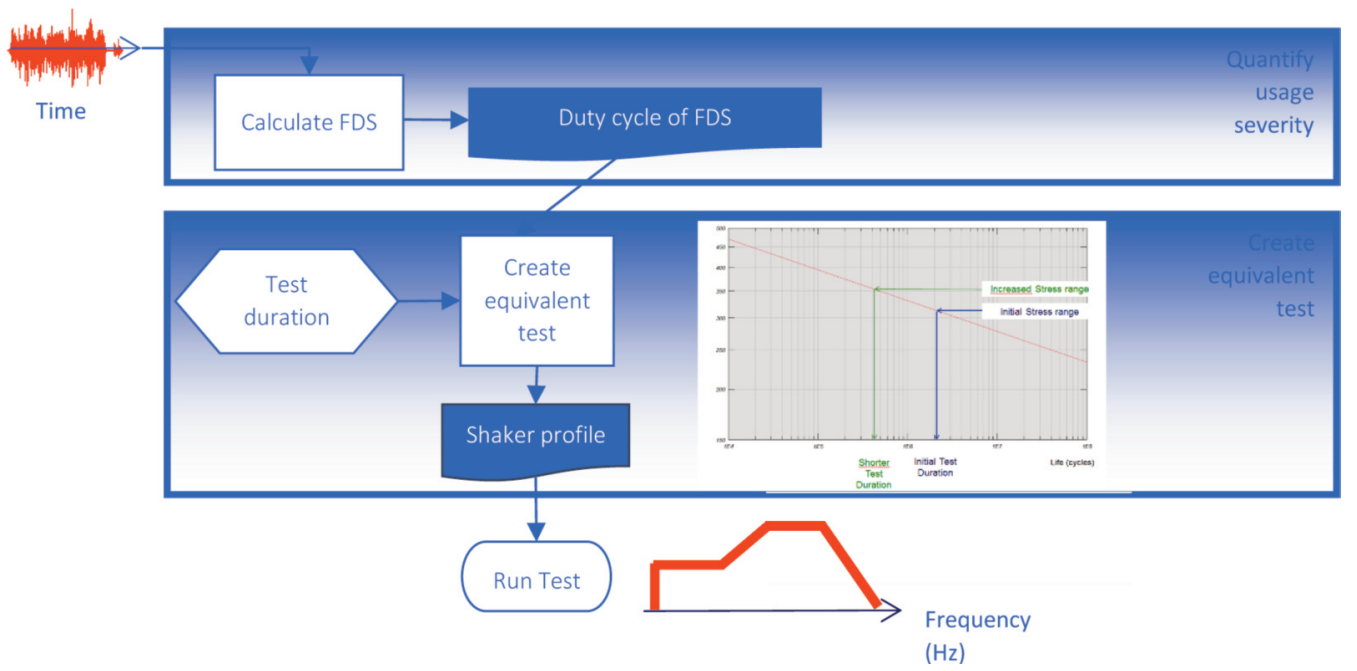

Fig. 8. Creation of Power Spectrum Density flowchart [4].

General assumptions introduce a single degree-of-freedom model, which enables, for comparison purposes, fatigue to be calculated from acceleration. Linear damage accumulation is described with Miner's Rule, which uses the linear combination of several events for the mission profile. Those steps create a Fatigue Damage Spectrum (FDS) from acceleration measurements, for one single event or a combination of several events, which describes the full mission profile. Once the duty cycle of FDS's is obtained, an inverse operation is performed to get PSD, where we specify the test duration. When increasing the amplitude, we can maintain the damage constant and obtain a shorter test duration.

\section{ANALYSIS: HOW TO DEFINE A VIRTUAL SHAKER TEST?}

Once the foundations of the FE model are established as regards geometry and loading, fatigue analysis can be performed in time domain or frequency domain.

The virtual shaker test in frequency domain uses FE harmonic analysis with base unitary acceleration and PSD loading subjected to exposure duration. Statistical rainflow (Steinberg, Narrow Band, Lalanne or Dirlik) is obtained from spectral 
moments, and a linear damage accumulation calculation is done at the required exposure duration [6].

The latest models' refinements integrate plasticity correction with EN analysis to give a better description of the stress field close to hot spots. It is also possible to include the sigma clipping introduced by some shaker controller.
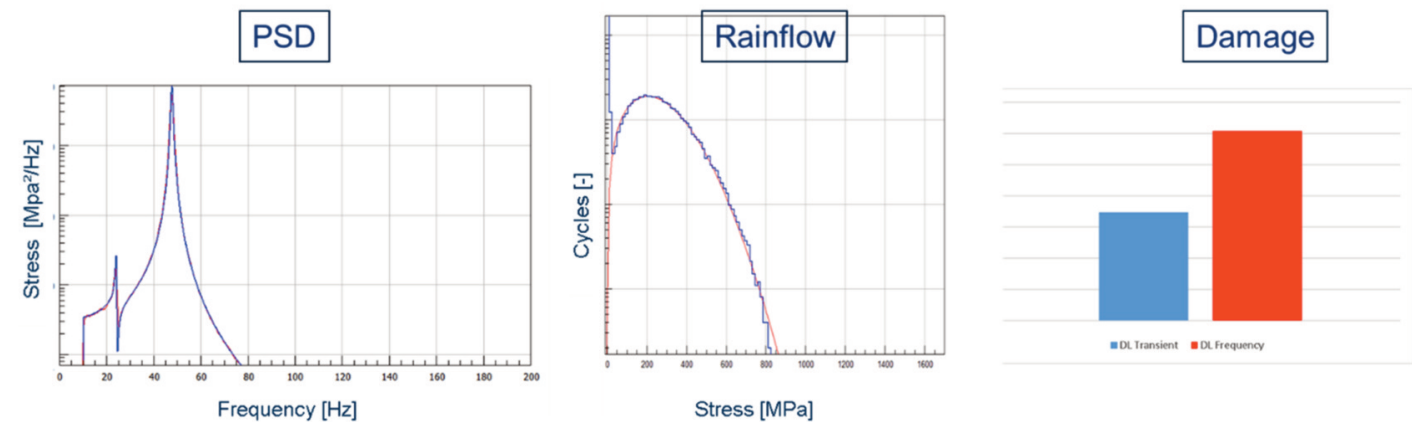

Fig. 9. Example of FE Vibration analysis output (PSD of stress), rainflow and damage (blue: time domain, red: frequency domain).

Same outputs are available in the time domain approach, PSD of stress and rainflow. Results are similar to those obtained in the frequency domain. Small differences for high stresses classes result in a more conservative value when the frequency domain approach is used, the reason being that the asymptotic rainflow is better described. The time domain analysis is more intuitive and may address non-stationary events. The frequency domain is computationally more efficient, as no influence of exposure duration applies on computational analysis time, more precise, as a better description of rainflow is achieved, and, finally the latest refinements in analysis lead to more accurate results.

\section{CONCLUSION}

This paper shows the various steps needed to build a digital twin for fatigue analysis. Firstly, the importance of the FE/test correlation is demonstrated, where using virtual sensors and measurements from strain gages and accelerometers brings a real benefit. A framework for generating a PSD mission profile from a set of accelerometers measurements is then established, giving first order magnitude input for analysis. Finally, a virtual shaker test is built, in both the time domain and frequency domain, with finely tuned assumptions to make the simulation analysis results closer to reality.

\section{REFERENCES}

[1] Conference «HBM on Tour », « Réduire la durée de vos essais ? « Réel et Virtuel: Une journée pour vous convaincre », Loic Guérin (HBK), Henri Gérenton (HBK), Amaury CHABOD (HBK).

[2] Gieck, K. and Bendit, G. (1997). Formulaire Technique, 10e édition revue et complétée. Dunod, France. 
[3] White paper nCode, “Accelerated Vibration Testing based on Fatigue Damage Spectra", Dr Andrew Halfpenny.

[4] Conference nCode, "The Role of Vibration in Fatigue", 5th October 2017, Hotel Novotel Part Dieu, Lyon.

[5] Meirovitch, L. (2001). Fundamentals of Vibrations, McGraw-Hill International Edition, Mechanical Engineering Series, p. 400.

[6] DesignLife Theory Guide, paragraphs 3.6 and 5.3. 